\title{
Estudo comparativo da resistência de união metal/resina entre duas técnicas de colagem de tubos ortodônticos
}

\section{Comparative study of metal/resin union resistance in two orthodontic bonding techniques of tubes}

\section{Estudio comparativo de la resistencia a la unión de metal/resina en dos técnicas de unión ortodónica de tubos}

\author{
Eduardo Efraín Vivanco Carpio ${ }^{1}$ \\ Luiz Eduardo Alessio Junior iD \\ 1 dreduvivancoc@hotmail.com \\ Endereço para correspondência: \\ Luiz Eduardo Alessio Junior \\ Rua Henrique Savi, 3-62 \\ 17012-205 - Bauru - São Paulo - Brasil \\ E-mail: lui.alessio@gmail.com
}

Recebido: 13.08 .2020

Modificado: 28.08.2020

Aceito: 11.09.2020

\begin{abstract}
RESUMO
Inúmeros estudos demonstram que os adesivos têm excelente união ao esmalte, portanto o objetivo deste estudo é avaliar a união na interface entre o metal do tubo ortodôntico e a resina. Neste estudo da resistência ao cisalhamento será utilizado resina Transbond XT 3MTM em 40 tubos ortodônticos para molares inferiores simples slot 0.022 ", que serão divididos em dois grupos: 20 tubos ortodônticos para o Grupo Controle (GC) e 20 tubos ortodônticos para o Grupo Experimental (GP). No GC será adicionado uma camada de resina de cimentação ortodôntica de $1 \mathrm{~mm}$, seguido de fotopolimerização com 0 mm de distância por 10 segundos para evitar a contração máxima da resina, mais 20 segundos de fotopolimerização contínuo, usando uma lâmpada de $700 \mathrm{MW}$, e uma segunda camada de $2 \mathrm{~mm}$ retangular à forma da base do tubo ortodôntico para a aplicação do teste de cisalhamento. Para o Grupo Experimental (GP), a malha metálica da base do tubo foi condicionada com a aplicação de micro abrasão com jato de óxido de alumínio de 50 mícron, por 8 segundos a uma distância de 2 a 3 mm através da superfície da malha do tubo, a uma pressão de ar de $90 \mathrm{~Pa}$, controlado por um barômetro de ar comprimido. Logo após foi realizada a remoção das impurezas do óxido de alumínio usando uma seringa de ar e água da unidade odontológica. Na superfície limpa foi aplicado o sistema Primer Adesivo Transbond XT $\mathrm{XT}^{\mathrm{TM}}$ com fotopolimerização por 10 segundos com luz LED contínua a 0mm, em seguida foi colocada a resina Transbond XT ${ }^{\mathrm{TM}}$ em 3 camadas de $1 \mathrm{~mm}$, com 10 segundos de fotopolimerização a 0 mm. Todos os corpos de prova foram marcados e levados para a máquina de testes universal onde a força será aplicada à superfície da resina presa à malha do tubo ortodôntico, a uma distância de $0.8 \mathrm{~mm}$ da malha. São valores numéricos, portanto, o Teste t de Student para amostras independentes foi selecionado. Com base nos resultados obtidos, afirma-se que o Grupo Controle GC e o Grupo Experimental com o condicionamento de jato de óxido de alumínio às bases dos tubos ortodônticos, não apresentam diferença estatisticamente significante.
\end{abstract}

PALAVRAS-CHAVE: Ortodontia. Resinas. Materiais dentários. 


\begin{abstract}
Numerous studies demonstrate that adhesives have excellent bonding to enamel, so the objective of this study is to evaluate the bonding at the interface between the metal of the orthodontic tube and the resin. Materials and Methods: In this shear strength study, Transbond XT 3M $\mathrm{M}^{\mathrm{TM}}$ resin will be used in 40 orthodontic tubes for simple 0.022 "lower molars, which will be divided into two groups: 20 orthodontic tubes for the Control Group (GC) and 20 orthodontic tubes for the Experimental Group (GP). In the $\mathrm{GC}$, a layer of $1 \mathrm{~mm}$ orthodontic cementation resin will be added, followed by light curing with $0 \mathrm{~mm}$ distance for 10 seconds to avoid maximum contraction of the resin, plus 20 seconds of continuous light curing, using a $700 \mathrm{MW}$ lamp, and a second layer of $2 \mathrm{~mm}$ rectangular to the shape of the base of the orthodontic tube for the application of the shear test. For the Experimental Group (GP), the metallic mesh at the base of the tube was conditioned with the application of micro abrasion with a 50 micron aluminum oxide jet, for 8 seconds at a distance of 2 to $3 \mathrm{~mm}$ across the surface of the mesh. tube, at an air pressure of $90 \mathrm{~Pa}$, controlled by a compressed air barometer. Immediately afterwards, aluminum oxide impurities were removed using an air and water syringe from the dental unit. On the clean surface, the Transbond XT ${ }^{\mathrm{TM}}$ Primer Adhesive system was applied with light curing for 10 seconds with $0 \mathrm{~mm}$ continuous LED light, then the Transbond XT'M resin was placed in 3 layers of $1 \mathrm{~mm}$, with 10 seconds of light curing at $0 \mathrm{~mm}$. All specimens were marked and taken to the universal testing machine where the force will be applied to the resin surface attached to the mesh of the orthodontic tube, at a distance of $0.8 \mathrm{~mm}$ from the mesh. They are numerical values, so the Student's t-test for independent samples was selected. Based on the results obtained, it is stated that the Control GC Group and the Experimental Group with the conditioning of aluminum oxide jet to the bases of orthodontic tubes, do not present a statistically significant difference.
\end{abstract}

KEYWORDS: Orthodontics. Resins. Dental materials.

\title{
RESUMEN
}

Numerosos estudios han demostrado que los adhesivos tienen una excelente unión con el esmalte, por lo que el objetivo de este estudio es evaluar la unión en la interfaz entre el tubo de ortodoncia metal y resina. Materiales y métodos: En este estudio de resistencia al cizallamiento, Transbond XT $3 \mathrm{M}^{\mathrm{TM}}$ resina se utilizará en 40 tubos de ortodoncia para ranuras simples de molares inferiores 0.022 ", que se dividirán en dos grupos: 20 tubos de ortodoncia para el Grupo de Control (CG) y 20 tubos ortodónticos para el Grupo Experimental (GP). En el CG se añadirá una capa de resina de cementación ortodoncia de $1 \mathrm{~mm}$, seguida de un curado ligero con $0 \mathrm{~mm}$ de distancia durante 10 segundos para evitar la máxima contracción de la resina, más 20 segundos de curado continuo de la luz, utilizando una lámpara de $700 \mathrm{MW}$, y una segunda capa de $2 \mathrm{~mm}$ rectangular a la forma de la base del tubo ortodóntico para la aplicación de la prueba de cizallamiento. Para el Grupo Experimental (GP), la malla metálica de la base del tubo fue acondicionada con la aplicación de micro abrasión con chorro de óxido de aluminio de 50 micras, durante 8 segundos a una distancia de 2 a $3 \mathrm{~mm}$ a través de la superficie de la malla del tubo, a una presión de aire de $90 \mathrm{~Pa}$, controlada por un barómetro de aire comprimido. Inmediatamente después, las impurezas del óxido de aluminio lo estaban retirando, utilizando una jeringa de aire y agua de la unidad dental. En la superficie limpia, se aplicó el sistema Transbond XT Adhesivo Primer ${ }^{\mathrm{TM}}$ con curado ligero durante 10 segundos con luz LED continua a $0 \mathrm{~mm}$, luego el Transbond XT ${ }^{\mathrm{TM}}$ resina se colocó en 3 capas de $1 \mathrm{~mm}$, con 10 segundos de curado de luz de $0 \mathrm{~mm}$. Todas las muestras han sido marcadas y llevadas a la máquina de ensayo universal donde la fuerza se aplicará a la superficie de resina unida a la malla del tubo ortodóntico a una distancia de 0.8 $\mathrm{mm}$ de la malla. Estos son valores numéricos, por lo que se ha seleccionado la prueba $\mathrm{T}$ del estudiante para muestras independientes. Sobre la base de los resultados obtenidos, se afirma que el Grupo de Control GC y el Grupo Experimental con el acondicionamiento de chorro de óxido de aluminio a las bases de los tubos de ortodoncia, no presentan diferencia estadísticamente significativa.

PALABRAS CLAVE: Ortodoncia. Resinas. Materiales dentales. 


\section{INTRODUÇÃO}

Dentro das dificuldades de manter os diferentes acessórios ortodônticos fixos, temos os tubos dos molares inferiores, devido ao impacto que eles sofrem às forças da mastigação que levam ao desprendimento destes, produzindo atrasos no tratamento ortodôntico'. As forças de mastigação em adultos jovens saudáveis têm sido relatados um valor médio de $727 \mathrm{~N}$, em crianças com dentição permanente e oclusão normal um valor de $425 \mathrm{~N}$, em mulheres jovens com doença periodontal um valor de $370 \mathrm{~N}$, em crianças saudáveis com dentição decídua um valor de $186.20 \mathrm{~N}$, a assimetria de carga tem o potencial de causar danos aos dentes, e essas forças de mastigação são transmitidas a receptores que percebem mudanças de força e agem para causar uma resposta inibitória dos músculos da mandíbula e, assim, prevenir o excesso de atividade muscular e a força de mastigação, em idosos com próteses parciais removíveis ou próteses totais um valor de $181 \mathrm{~N} \mathrm{e}$ em crianças na dentadura mista e mordida cruzada um valor de 114 N. A relação entre variáveis corporais e força de mastigação, nos estágios iniciais da vida, depende de fatores mais complexos e que essa relação é posteriormente aumentada a partir da puberdade com o aumento do índice de corporal. Em relação aos adultos, há a aceitação de que a força mastigatória massa é maior em homens do que em mulheres; tal é a força dessa premissa que, em um registro de força de mastigação durante a fase de manutenção da terapia periodontal, foi predeterminado que a alta força mastigatória seria diagnosticada com homens mostrando mais de $500 \mathrm{~N}$ e mulheres mostrando mais de $370 \mathrm{~N}$. A explicação para o acima citado é que os músculos masseteres dos homens têm fibras tipo 2 com áreas e diâmetros maiores do que as mulheres ${ }^{2}$.

O desprendimento de um tubo ortodôntico está relacionado à força de mastigação de cada paciente, por isso é escolhido bandar os dentes com as bandas préfabricadas para molares e com o tubo para soldagem desde o início do tratamento para evitar este inconveniente ${ }^{3}$. No início deste século muitos têm sido os avanços da ciência e da tecnologia na evolução dos materiais odontológicos com grandes benefícios para cada uma das áreas da odontologia, simplificando as atividades clínicas para os diversos tratamentos, atualmente em ortodontia há também a grande evolução e desenvolvimento dos materiais que facilitam a instalação de diversos dispositivos ortodônticos, com isso dependemos de diferentes cimentos dentários, ionômeros e resinas sempre buscando a fixação e estabilidade dos acessórios ortodônticos no ambiente oral agressivo, mas ainda há falha em certos acessórios como aqueles que são a razão para este estudo.

A adesão direta dos tubos ortodônticos ao esmalte elimina a necessidade de bandas, foi um sonho por muitos anos, antes de se tornar uma técnica de rotina nos anos 80. A adesão baseia-se na ligação mecânica de um adesivo às irregularidades do esmalte e superfície dos dentes, condicionado por ácido fosfórico de $37 \%$ e as uniões mecânicas formadas nas bases dos tubos e bráquetes ortodônticos. Portanto, para obter resultados satisfatórios na adesão ortodôntica, deve-se prestar muita atenção a três componentes do sistema: a superfície do dente e sua preparação, o desenho da base dos tubos e bráquetes e o próprio material adesivo. A base de um bráquete e tubo deve ser fabricada de tal forma que uma interconexão mecânica entre o material de união e a base do tubo ortodôntico possa ser alcançada. As uniões químicas às vezes são muito fortes e criam problemas durante a remoções dos aparelhos; por causa disso, a retenção mecânica é atualmente preferida para bráquetes metálicos e cerâmicos. Um bom material adesivo deve atender a uma série de critérios: deve ser dimensionalmente estável; deve ser fluido o suficiente para penetrar a superfície do esmalte; ter uma excelente resistência, e deve ser fácil de usar clinicamente. Atualmente, os materiais adesivos mais utilizados são resinas acrílicas (Bis-GMA). Estão disponíveis em diferentes formulações, em apresentação química ou fotopolimerizável ${ }^{3}$.

Apesar do avanço dos materiais e de várias modificações nos desenhos da base dos tubos ortodônticos e bráquetes metálicos, para melhorar a retenção mecânica, observa-se que há falhas na união da resina com a base metálica, com falta de resistência e durabilidade à agressão que o aparelho em geral recebe durante a mastigação. Para melhorar o acima citado, este estudo avaliou o condicionamento com jato de óxido de alumínio na base de tubos ortodônticos correspondentes aos primeiros molares inferiores, para observar se a micro abrasão é relevante, para melhorar ou aumentar a união da resina na base do tubo.

\section{MATERIAL E MÉTODOS}

Para o estudo serão utilizados: 40 tubos ortodônticos simples para molares inferiores slot 0,022 " com gancho prescrição Roth (Morelli); fio de aço ortodôntico 0,019 " X 0,025"; 40 tubos metálicos quadrados de aço inoxidável 2 $\mathrm{cm}$ X $2 \mathrm{~cm}$; cola Super Bond; resina acrílica autopolimerizável para identificar os grupos, cor de cristal para grupo controle e cor amarelo para grupo experimental; resina ortodôntica para colagem Transbond XT ${ }^{\mathrm{TM}}(3 \mathrm{M})$ fotopolimerizável; lâmpada de luz LED Radii Plus; medidor de intensidade de energia leve para verificar 700 MW; cronômetro (Microetcher Gun); óxido de alumínio 50 $\mu \mathrm{m}$; barômetro e máquina de teste universal da Universidade Politécnica Nacional do Equador.

Para realizar este estudo foi realizado uma metodologia 
modificada e similar a outros estudos para cisalhamento. Este estudo utilizou 40 cubos metálicos de aço inoxidável de $2 \mathrm{~cm} \mathrm{X} 2 \mathrm{~cm}$ que irá fixar cada um dos tubos ortodônticos em seu slot por meio de um fio de aço ortodôntico 0.019 " X 0.025" colado com Super Bond, as bordas cubo de aço.

Para dar estabilidade, o tubo foi preenchido com acrílico de autopolimerização, deixando sua base com a malha totalmente horizontal e livre. Os 40 tubos, foram divididos em dois grupos, Grupo Controle (GC) (Figura 1), marcado com a cor cristal e Grupo Experimental (GP) marcado com amarelo da resina acrílica autopolimerizável.

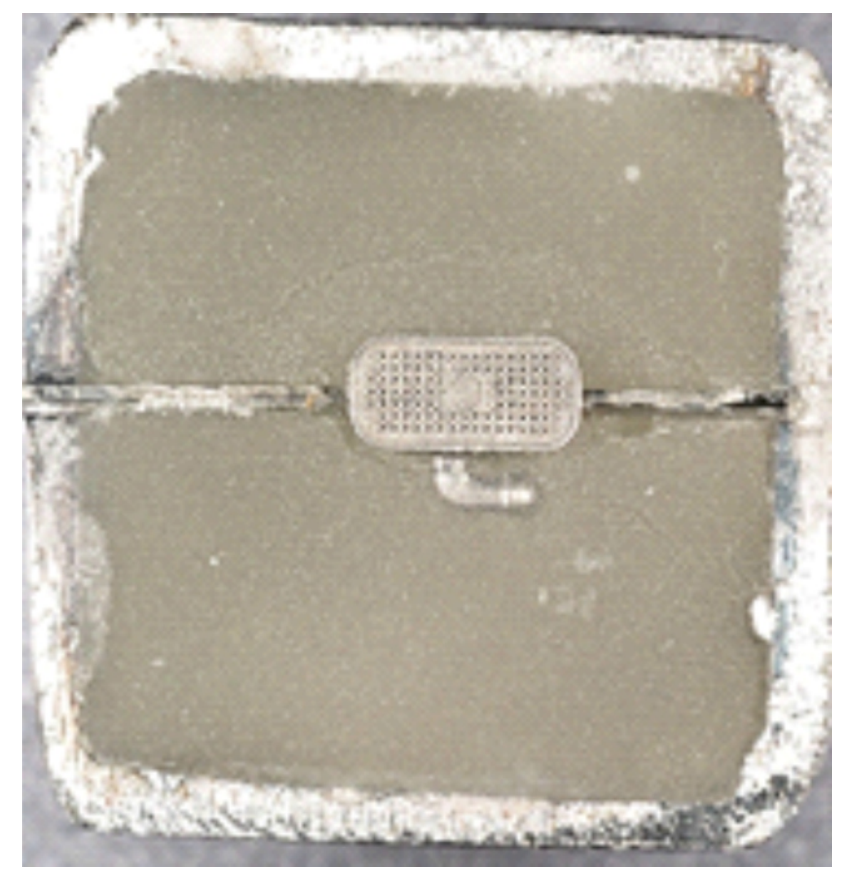

Figura 1 - Tubo ortodôntico para Grupo Controle.

Para este estudo também foi preparada a base do tubo ortodôntico, que foi realizado com camadas de resina ortodôntica, onde a força para o teste de cisalhamento será aplicada, o bloco de resina tem em média $3 \mathrm{~mm}$ de espessura, da superfície da resina até a base do tubo ortodôntico, uma canaleta é feita com a forma de ponta, com um disco metálico de baixa rotação uma distância de $0.8 \mathrm{~mm}$, medido equidistante à área vestibular do tubo, superfície na qual o alimento impacta ou a pressão de mastigação ocorre pelos dentes antagônicos.

\section{Grupo Controle (GC)}

Em vinte corpos de prova com acrílico autopolimerizável da cor cristal para identificar o grupo controle, sem o condicionamento da mala da base do bráquete foi aplicada a resina de colagem ortodôntica Transbond XT ${ }^{\mathrm{TM}}$, em camada para se obter um retângulo de $3 \mathrm{~mm}$ de resina, onde será aplicada a força de cisalhamento.

A primeira camada de $1 \mathrm{~mm}$ resina foi aplicada diretamente na malha com uma pequena porção de resina compactada de maneira firme e estável contra a malha, preenchendo todos os espaços da malha e cobrindo a toda a superfície metálica, após a aplicação da resina foi realizada a fotopolimerização por 10 segundos com luz intermitente à $0 \mathrm{~mm}$ de distância, para evitar o máximo de contração e evitar que produza falhas micro mecânicas e falhas de adesão na estrutura de retenção da malha do tubo ortodôntico.

Em seguida, uma segunda camada de resina é aplicada, formando um retângulo, que está nos limites da base do tubo ortodôntico, aplicado manualmente e modelado até obter um retângulo de $1 \mathrm{~mm}$ de espessura e fotopolimerizamos por 10 segundos com luz continua à $0 \mathrm{~mm}$ de distância.

Colocamos outra porção de resina para formar a terceira camada de resina de $1 \mathrm{~mm}$ e continuamos modelando um retângulo sobre a primeira e segunda camada de resina e fotopolimerizamos por 20 segundos a 0 $\mathrm{mm}$ de distância, neste grupo não se aplicará o Primer Adesivo Transbond $\mathrm{Xt}^{\mathrm{TM}}$.

\section{Grupo Experimental (GP)}

Nas malhas dos tubos do grupo experimental (GP) foi aplicado micro abrasão utilizando pistola Microetcher com óxido de alumínio de 50 ?m durante 8 segundos a $5 \mathrm{~mm}$ de distância da malha do tubo ortodôntico a una pressão de ar de $90 \mathrm{~Pa}$.

Após a aplicação do jato de óxido de alumínio, os tubos foram lavados com água destilada (seringa triplex da unidade do equipo dentário) e secados com ar comprimido até observar a opacidade do metal deixado pelo jateamento.

Aplicação do Primer Adesivo Transbond XT'TM fotopolimerizável no tubo com aplicador e com ar foi removido os excessos para deixar uma camada uniforme e fina que hidrate as microabrasões, seguido de fotopolimerização com potência de luz de $700 \mathrm{MW}$ por 20 segundos a $0 \mathrm{~mm}$ de distância.

Aplicação da resina para colagem ortodôntica Transbond XT ${ }^{\mathrm{TM}}$ em 3 camadas de $1 \mathrm{~mm}$, a primeira compactando sobre a malha de maneira firme e estável e fotopolimerização com luz intermitente por 10 segundos mais 20 segundos de fotopolimerização a $0 \mathrm{~mm}$ e a segunda e terceira camada formando um retângulo sobre a malha do tubo ortodôntico seguido de fotopolimerização recomendada pelo fabricante (20 segundos) (Figura 2). 


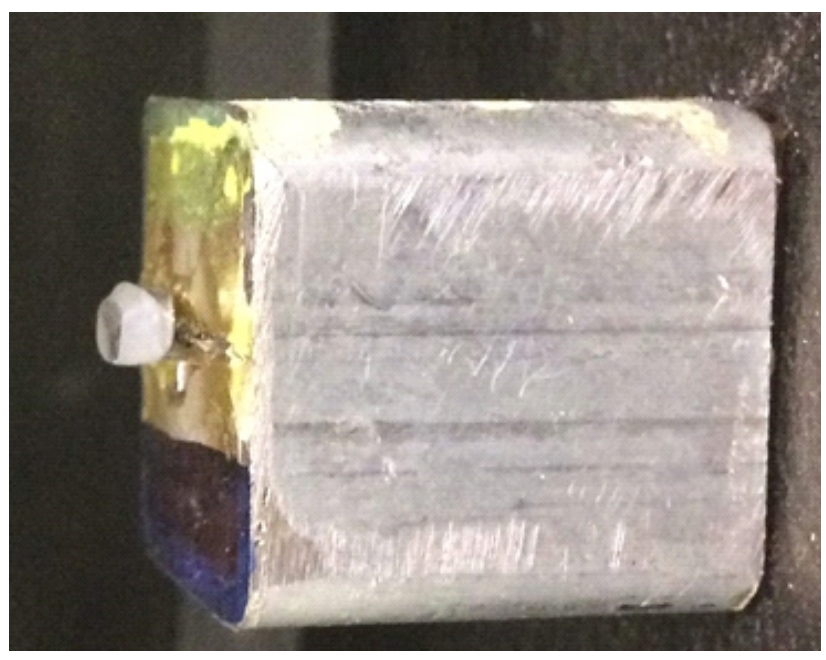

Figura 2 - Grupo Experimental com três camadas de resina.

Após 8 dias de preparação dos corpos de prova, esperando que a resina fotopolimerizável complete sua resistência absoluta para ser realizado o teste de cisalhamento na máquina de ensaios. Em todos os corpos de prova uma canaleta foi realizada, com um disco metálico de baixa rotação a uma distância de $0.8 \mathrm{~mm}$ à área vestibular do tubo. A força de cisalhamento foi aplicada no bloco de resina a una distância equivalente as forças de impacto mastigatório, $0.8 \mathrm{~mm}$. A uma velocidade de 2 minutos por milímetro. Até o desprendimento da resina.

\section{Análise Estatística}

Para a realização do teste estatístico, em um estudo transversal, pois dois grupos são analisados e comparados como variável fixa ao mesmo tempo e a variável aleatória são valores numéricos, foi selecionado o teste $t$ de Student para amostras independentes. Com os resultados obtidos, registrou-se em unidades Mpa para o teste de cisalhamento. Levando-se em conta sua carga máxima e carga mínima registrada na área de incidência do tubo ortodôntico, em cada um dos grupos (GC e GP). Um dos grupos não se comporta com uma distribuição normal, portanto, um teste não paramétrico U de Mann-Withney foi exigido neste caso para verificar se as medidas são exatamente as mesmas ou não.

\section{RESULTADOS}

Os dados do teste de cisalhamento de cada grupo foram inseridos em um banco de dados no programa SPSS da versão 22 da IBM para realizar cálculos sobre estatísticas descritivas e inferenciais. Como se pode observar, não existem valores atípicos para todos os grupos, de modo que a estatística descritiva poderá, portanto, ser analisada de acordo com os valores do teste de cisalhamento (Tabela 1).

Tabela 1 - Resultados estatísticos descritivos.

\begin{tabular}{l|l|l|l|l|l}
\hline & Tipo & $\mathrm{N}$ & Média & $\begin{array}{l}\text { Desvio } \\
\text { padrão }\end{array}$ & $\begin{array}{l}\text { Erro padrão } \\
\text { médio }\end{array}$ \\
\hline $\begin{array}{l}\text { Teste de } \\
\text { cisalhamento }\end{array}$ & $\begin{array}{l}\text { Grupo } \\
\text { C }\end{array}$ & 20 & 13.4390 & 4.14080 & .92591 \\
\cline { 2 - 6 } & $\begin{array}{l}\text { Grupo } \\
\text { P }\end{array}$ & 20 & 13.9030 & 4.38935 & .98149 \\
\hline
\end{tabular}

A Tabela 1 mostra as estatísticas sumárias do Grupo Controle GC e do Grupo Experimental que incluem medidas de tendência central.

Grupo GC: A amostra tem uma média de 13.44Mpa, um desvio padrão de 4.14Mpa.

Grupo GP: A amostra tem uma média de 13.90Mpa, um desvio padrão de 4.39Mpa.

De acordo com a análise é um estudo transversal porque dois grupos estão sendo analisados como uma variável fixa ao mesmo tempo e a variável aleatória são valores numéricos, portanto, o Teste $\mathrm{t}$ de Student para amostras independentes foi selecionado.

Antes de realizar o teste $\mathrm{t}$ de Student, é necessário começar realizando um teste de normalidade (Figura 3). Deve-se verificar se a variável aleatória é normalmente distribuída, para isso o teste Shapiro-Wilk é escolhido porque é eficaz quando o tamanho da amostra é menor ou igual a 30.

\begin{tabular}{|l|l|l|l|l|l|l|l|}
\hline \multirow{2}{*}{ Tipo } & \multicolumn{5}{|l|}{ Kolmogorov-Smirnov } & \multicolumn{3}{l|}{ Shapiro-Wilk } \\
\cline { 4 - 8 } & & Estatística & gl & Sig. & Estatística & gl & Sig. \\
\hline Teste de Cisalhamento Grupo C & .125 & 20 & $200 *$ & .951 & 20 & .383 \\
& Grupo P & .202 & 20 & .032 & .886 & 20 & .023 \\
\hline
\end{tabular}

a. Lilliefors correção de significado.

Figura 3 - Teste de normalidade.

$\mathrm{O}$ resultado do teste de normalidade demonstra que o Grupo Controle GC vêm de uma população com distribuição normal (0.383), pois excedem o nível de significância imposto por 0,05, e o Grupo Experimental GP (0.023) é inferior a 0.05 , portanto, não vem de uma distribuição normal. A variável do teste de cisalhamento para o grupo GC se comporta normalmente (Ho), enquanto o grupo GP não se comporta normalmente (Ha)

Uma vez que um dos grupos não se comportam com uma distribuição normal, é necessário neste caso realizar um teste não paramétrico U Mann-Whitney. Em seguida na Figura 4 será demonstrada a análise U de Mann-Whitney para verificar se as médias são estaticamente iguais ou não. Concluiu-se que não há diferença significativa entre o teste de cisalhamento entre as amostras do Grupo GC e do Grupo GP, valor de $\mathrm{P}=1.000$. 


\begin{tabular}{|lr|}
\hline N total & 40 \\
\hline U de Mann-Whitney & 200,000 \\
\hline W de Wilcoxon & 410,000 \\
\hline Estadistico de contraste & 200,000 \\
\hline Error estándar & 36,968 \\
\hline $\begin{array}{l}\text { Estadistico de contraste } \\
\text { estandarizado }\end{array}$ &, 000 \\
\hline $\begin{array}{l}\text { Significación asintótica (prueba } \\
\text { bilateral) }\end{array}$ & 1,000 \\
\hline $\begin{array}{l}\text { Significación exacta (prueba } \\
\text { bilateral) }\end{array}$ & 1,000 \\
\hline
\end{tabular}

Figura 4 - Teste amostral independente.

\section{DISCUSSÃO}

Os tubos ortodônticos são muito utilizados na ortodontia contemporânea, a adesão direta dos tubos nos molares é uma prática comum, ou temos a opção de bandagem dos molares e soldagem de tubos a banda, devido ao inconveniente do deslocamento de tubos colados nos molares inferiores. As vantagens da colagem direta são: redução do tempo de consulta para montar o aparelho ortodôntico fixo, favorece a manutenção da saúde periodontal, elimina os espaços causados pelas bandas (diastemas), facilita a detecção de cavidades e cáries, proporciona uma melhor estética e facilidade de manuseio ${ }^{4}$.

Um estudo nos Estados Unidos mostrou que apenas $30 \%$ dos ortodontistas costumam colar os tubos dos molares. Este fato provavelmente está relacionado com a alta taxa de falha clínica deste procedimento. No entanto, não se dá ênfase à literatura sobre bandas e tubos soltos, com exceção de um estudo que comparou a colagem direta dos molares com a utilização de bandas. Os autores avaliaram os molares em adolescentes e adultos, observando que molares inferiores com bandas em ambas as faixas etárias apresentaram perda significativamente maior de inserção periodontal durante o tratamento do que os molares inferiores com tubos colados ${ }^{4}$.

Os avanços nas resinas ajudaram a reduzir a taxa de falha dos procedimentos ortodônticos. Hoje, parece mais importante melhorar os procedimentos clínicos operacionais do que aumentar a resistência retentiva das resinas atualmente em uso. Vários estudos têm avaliado que as resinas têm excelente adesão ao esmalte. O sistema adesivo Transbond XT foi selecionado para este estudo devido aos dados comparativos existentes indicando melhores resultados obtidos na adesão ortodôntica do que com outros sistemas adesivos.

O estudo que demonstra a melhor opção de colagem para tubos ortodônticos é o sistema adesivo Transbond $\mathrm{XT}^{\mathrm{TM}}$ comparou a resistência dos bráquetes ao esmalte após 60 minutos e 24 horas de fixação com dois sistemas de adesão diferentes. Foram utilizados 60 pré-molares divididos em 4 grupos de 15 amostras cada, Transbond XT Plus $^{\mathrm{TM}}$ (grupos 3 e 4) e Transbond XT ${ }^{\mathrm{TM}}$ (grupos 1 e 2). As amostras foram submetidas as forças tangenciais da máquina Universal Instron com uma velocidade de carga de $1.0 \mathrm{~mm} / \mathrm{min}$ para obter a resistência ao deslocamento. Após a realização da análise estatística ANOVA, mostrou-se que não há diferenças estatísticas estatisticamente significativas na força para o desempenho entre Transbond XT ${ }^{\mathrm{TM}}$ em 24 horas (média 6.8 Mpa) e Transbond XT ${ }^{\mathrm{TM}}$ Plus em 24 horas (média 6.1 Mpa).

Com base nos resultados obtidos, afirma-se que o Grupo Controle GC e o Grupo Experimental com o condicionamento de jato de óxido de alumínio às bases dos tubos ortodônticos, não apresentam diferença estatisticamente significante, é importante mencionar que o maior valor de desvio padrão (Tabela 1) foi levemente maior para o Grupo Experimental GE. Isso pode ser atribuído ao processo de condicionamento na malha da base do tubo; embora o objetivo seja realizar com métodos padronizados, a micro abrasão foi realizada manualmente. As malhas dos tubos novos condicionados, atendem aos requisitos adequados para cimentação direta ao dente, a amostra tem uma média de $13.90 \mathrm{Mpa}$, um desvio padrão de $4.39 \mathrm{Mpa}$, apresentando valores maiores, de acordo com as faixas de resistência ao cisalhamento ${ }^{6} 5.9$ a $7.8 \mathrm{Mpa}$.

A força de cisalhamento foi aplicada no bloco de resina a una distância equivalente as forças de impacto mastigatório, $0.8 \mathrm{~mm}$. A uma velocidade de 2 minutos por milímetro. Na revisão de literatura foram encontrados fatores que podem nos resultados do teste de força, no qual a área de contato da ponta da máquina de testes universal e o tubo ${ }^{7}$ a força aplicada entre as aletas e a base do bráquete pode diminuir o valor de resistência do que aplicar a força diretamente na base do bráquete que requer maior força ${ }^{8}$. Foram analisados em 3 grupos de 30 bráquetes, um dos grupos foi tratado por micro abrasão com partículas de pó de óxido de alumínio de 50 mícrons, sob uma pressão de 50 a 80 psi por 20 a 40 segundos, onde se conclui que a falha predominante ocorreu na interface resina e malha do bráquete. Por esta razão neste estudo se aplicou a força de cisalhamento diretamente sobre a resina a uma distância de 
$0.8 \mathrm{~mm}$ da malha do tubo, equivalente a distância onde se recebe as forças dos tubos quando estão na boca e não foi aplicada a força diretamente na interface resina e malha ${ }^{9}$.

Observou-se que ao realizar o protocolo de condicionamento recomendado em malhas de tubo ortodôntico (GP), a retenção aumentou ligeiramente, mas altera a distribuição normal nos valores, em relação ao grupo controle (GC) que manteve a estabilidade numérica.

Estes achados in vitro indicam que a aplicação de uma primeira camada de resina adesiva para cimentação de aparelhos ortodônticos após a micro abrasão com óxido de alumínio de $50 \mu \mathrm{m}$ em malhas de tubos ortodônticos novos não aumenta significativamente a força de união para o teste de cisalhamento. Novos estudos in vitro podem ser realizados em malhas metálicas com melhores características de retenção ou com micro abrasão com 25 , 75 e $100 \mathrm{~mm}$ de óxido de alumínio em um tempo de jateamento mais longo e comparar se há maior retenção das resinas para as malhas dos tubos. Esperava-se que resultados significativos de um aumento na retenção com tubos novos, possivelmente este método de micro abrasão e aplicação de adesivo na malha funciona na recimentação de tubos e bráquetes que estão há algum tempo no ambiente bucal, para reciclagem.

\section{CONCLUSÃO}

Com base nos resultados obtidos, afirma-se que o Grupo Controle GC e o Grupo Experimental, de acordo com o seu esforço de adesão ao teste de cisalhamento não apresentam diferença estatisticamente significante. Portanto, não é necessário fazer condicionamento com jato de óxido de alumínio às bases dos tubos ortodônticos.

\section{REFERÊNCIAS}

1. Moctezuma PEA. Fuerza de mordida: su importancia en la masticación, su medición y sus condicionantes clínicos. Parte I. Rev Asoc Dent Mex. 2012;69(2):53-7.

2. Koc D, Dogan A, Bek B. Bite force and influential factors on bite force measurements: a literature review. Eur J Dent. 2010;4(2):223.

3. Proffit WR. Ortodontia contemporânea. 4th ed. Barcelona: Elservier; 2009.

4. Boyd RL, Baumrind S. Periodontal considerations in the use of bonds or bands on molars in adolescents and adults. Angle Orthod. 1992;62(2):117-26.

5. Rodríguez Chávez JA, Barceló Santana FH, Borges Yáñez SA, Arenas Alatorre, JA. Comparación de la resistencia al desprendimiento de brackets entre dos sistemas adhesivos (SEP y
MIP Transbond) a 60 minutos y 24 horas. Rev Mex Ortod. 2013;1(1):38-44.

6. Reynolds IR. A review of direct orthodontic bonding. Brit J Orthod. 1975;2(3):171-8.

7. Benitez Díaz M, Kalach AM, Rivera Barrero, JR. Propiedades físicomecánicas y tipo de falla adhesiva del esmalte humano y bovino. Usta Salud. 2014;13(1):11-7.

8. Klocke A, Kahl-Nieke B. Effect of debonding force direction on orthodontic shear bond strength. Am J Orthod Dentofac Orthop. 2006;129(2):261-5.

9. Giraldo SL, Hernández C, Ospina A. Comparación de la resistencia adhesiva y tipo de falla de tres métodos de recondicionamiento de brackets para la recementación [thesis]. Bogotá: Fundación Universitaria CIEO-UniCIEO;2015. 